\title{
A propos des larves d'Ixodes pilosus et I. rubicundus
}

\author{
Par G. SENEVET et Ch. RIPERT *
}

Dans une précédente note (**) nous avons essayé de rassembler toutes les descriptions de larves de Tiques du genre Ixodes. Nous y avions omis celles d'Ixodes rubicundus, aussi Madame G. Theiler nous a-t-elle fait parvenir des larves d'Ix. rubicundus et d'Ix. pilosus provenant des collections du Zoological Survey of the Union of South Africa ; Ticks Survey. Cet envoi, dont nous tenons à la remercier ici, a rendu possible la description des larves de ces deux espèces et de leur chétotaxie qui est ici toute particulière. Avant nous, C. Clifford et M. Anastos (**) l'avaient déjà étudiée et nos recherches confirment leurs conclusions. Comme eux, nous serions tentés de placer $I x$. rubicundus et $I x$. pilosus dans un groupe spécial. Le tableau suivant montre en effet de façon fort nette les divergences entre les sous-genres Ixodes s. s., Pholeo-ixodes, Ixodiopsis, Exopalpiger, et le groupe du rubicundus. Certains des caractères de ce dernier groupe se retrouvent chez les Endopalpiger et les Sternalixodes.

\begin{tabular}{|c|c|c|c|}
\hline & $\begin{array}{c}\text { Soies } \\
\text { scutales }\end{array}$ & $\begin{array}{c}\text { Soies } \\
\text { supplémentaires }\end{array}$ & $\begin{array}{c}\text { Soies } \\
\text { prémarginales }\end{array}$ \\
\hline $\begin{array}{l}\text { Genre Ixodes en géné- } \\
\text { ral } \ldots \ldots \ldots \ldots \ldots \ldots \ldots\end{array}$ & 5 & 1 ou 0 & 4 \\
\hline Groupe du rubicundus. & 4 & 4 & 6 ou 7 \\
\hline
\end{tabular}

(*) Avec la collaboration technique de M. Bahbou. Laboratoires de Parasitologie des Facultés de Médecine d'Alger et de Paris.

(**) Amn. Parasitologie hum. et comp. (Paris), 1966. Cette note comprend une étude bibliographique complète des larves du genre Ixodes. 


\section{DESCRIPTION DES ESPECES}

\section{IXODES RUBICUNDUS NeUMANN, 1904}

Description d'après cinq exemplaires (fig. 1).

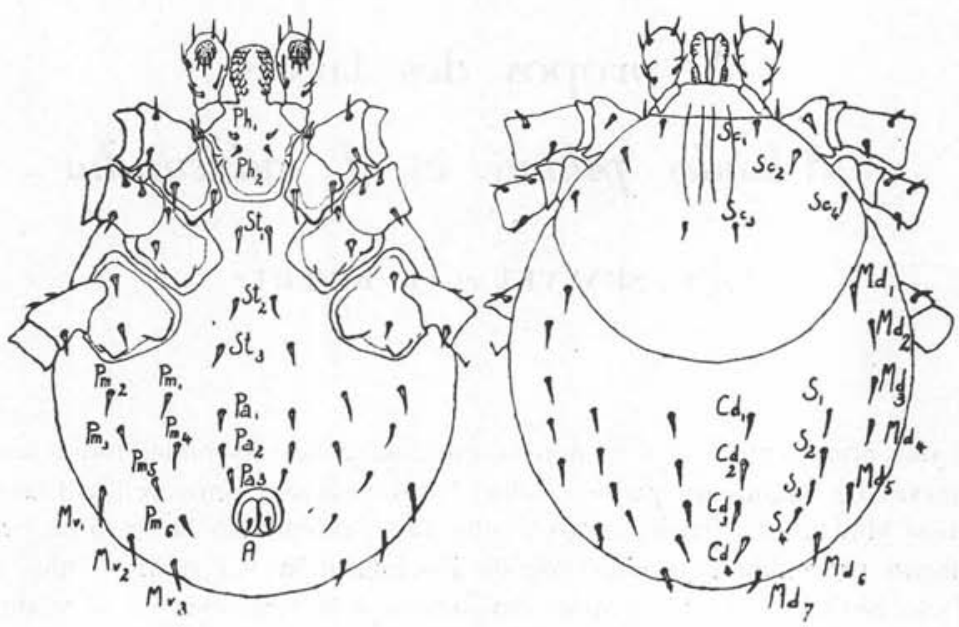

FIG. 1

\section{Corps.}

Subovale, sensiblement aussi long que large. Bord postérieur régulièrement arrondi ou portant des festons à peine marqués.

Longueur (de la base du capitulum à l'extrémité postérieure) : $0,375(0,353-$ $0,405)(*)$.

Largeur maxima : 0,375 $(0,328-0,409)$.

Rapport L/1: 1 .

\section{Capitulum.}

1. Base (face ventrale). Sensiblement trapézoïdale, nettement resserrée vers la moitié. Bord postérieur droit. Pas d'auricule.

Longueur (de la base à la moitié de l'hypostome) : $0,157(0,142-0,171)$.

Largeur (à l'insertion des palpes) : 0,092 (0,088-0,102).

Rapport L/1: 1,6.

2. Hypostome. Allongé, portant trois files de dents :

- file externe : $7 / 8$ dents longues et pointues ;

- file moyenne : $7 / 8$ dents, plus courtes et comme imbriquées ;

- file interne : $2 / 3$ dents, petites.

(*) Toutes les dimensions sont données en millimètres. 
Deux paires de soies posthypostomales insérées aux quatre angles d'un trapèze isocèle à petite base postérieure :

$$
\begin{aligned}
& \text { AA': } 0,056(0,042-0,080) \text {; } \\
& \text { PP' : } 0,052(0,042-0,063) \text {; } \\
& \text { AP : } 0,023(0,023-0,026) \text {. }
\end{aligned}
$$

3. Palpes. En massue, plutôt courts, à insertions largement écartées. Article I non hypertrophié. Articles II et III peu distincts. Largeur maximale du palpe située près de l'apex.

Longueur totale : $0,084(0,078-0,096)$;

Largeur maxima : $0,038(0,038-0,040)$.

Rapport L/1: 2,4 .

\section{Scutum.}

Assez régulièrement arrondi vers l'arrière.

Longueur : $0,244(0,225-0,270)$.

Largeur : 0,303- (0,277-0,328).

Quatre paires de soies scutales.

\section{Coxae et Tarse I.}

Coxa I. Un éperon postéro-externe. Trois soies.

Coxa II. Une crête postéro-externe. Deux soies.

Coxa III. Une crête postéro-externe. Une crête postéro-interne visible sur certains échantillons.

Longueur du tarse I : $0,191(0,185-0,200)$.

\section{Chétotaxie Générale.}

Face ventrale.

Trois paires de soies sternales. Longueur des soies: $\mathrm{St}_{1}: 0,027(0,021-0,035)$; St $_{2}$ : $0,022(0,021-0,023)$; $\mathrm{St}_{3}: 0,015(0,014-0,017)$.

Distances séparant $\mathrm{St}_{1}$ et $\mathrm{St}_{1}, \mathrm{St}_{1}$ et $\mathrm{St}_{3}$ : $\mathrm{St}_{1}-\mathrm{St}_{1}$ : $0,044(0,034-0,050), \mathrm{St}_{1}-\mathrm{St}_{3}$ : $0,110(0,102-0,119)$.

Trois paires de soies préanales: $\mathrm{Pa}_{1}, \mathrm{~Pa}_{2}, \mathrm{~Pa}_{3}$. 0,023).

Six paires de soies prémarginales : $\mathrm{Pm}_{1} \ldots \mathrm{Pm}^{6}$. Longueur de $\mathrm{Pm}_{6}$ : 0,021 (0,020-

Trois paires de soies marginoventrales: $\mathrm{Mv}_{1}, \mathrm{Mv}_{2}, \mathrm{Mv}_{3}$.

Face dorsale.

Quatre paires de soies centrodorsales: $\mathrm{Cd}_{1} \ldots \mathrm{Cd}_{4}$.

Quatre paires de soies supplémentaires: $S_{1} \ldots S_{4} . S_{1}: 0,025(0,021-0,028) ; S_{4}$ : $0,025(0,020-0,029)$.

Sept paires de soies marginodorsales: $\operatorname{Md}_{1} \ldots \mathrm{Md}_{7} . \mathrm{Md}_{1}: 0,020(0,019-0,021)$; $\mathrm{Md}_{7}$ : 0,011 (0,010-0,013). 


\section{IXODES PILOSUS Косн, 1844}

Description d'après six exemplaires (fig. 2).

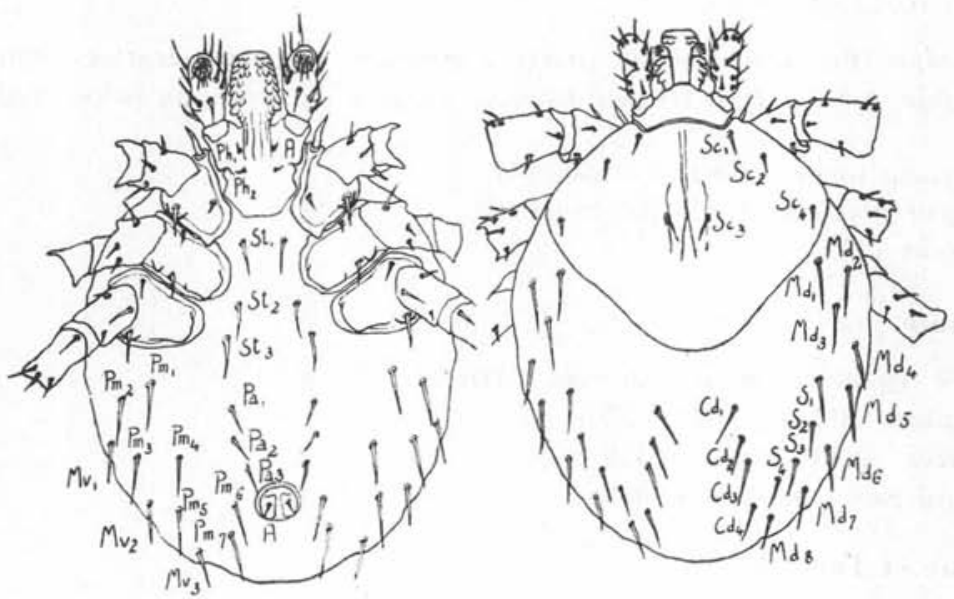

FIG. 2

\section{Corps.}

Régulièrement ovale. Festons postérieurs légèrement marqués. 0,451).

Longueur (de la base du capitulum à l'extrémité postérieure) : $0,425(0,410$ -

Largeur maxima: 0,421 (0,400-0,439).

Rapport L/1: 1,01 .

\section{Capitulum.}

1. Base (face ventrale). Quadrangulaire. Bord postérieur assez régulièrement arrondi en arrière. Une construction vers la partie médiane. Une auricule.

Longueur (de la base à l'extrémité de l'hypostome) : 0,195 (0,186-0,0220).

Largeur (à l'insertion des palpes) : 0,110 (0,103-0,116).

Rapport L/1 : 1,77.

2. Hypostome. Extrémité antérieure presque rectiligne. Trois file de dents:

- file externe : $7 / 8$ dents ;

- file moyenne: 7 dents ;

- file interne: 3 dents.

Deux paires de soies posthypostomales insérées aux quatre angles d'un trapèze presque rectangulaire : 
- AA': $0,055(0,052-0,059)$;

- PP' : $0,046(0,040-0,053)$;

- Ap : 0,026 (0,022-0,028).

3. Palpes. Article I non hypertrophié. Article II rétréci dans sa partie basale (sa largeur à ce niveau la moitié de celle de l'apex), portant une légère excroissance externe à la base. Limite entre les articles II et III imprécise.

Longueur : $0,110(0,099-0,120)$.

Largeur : $0,317(0,031-0,037)$.

Rapport L/1 3,3.

\section{Seutum.}

Bord postérolatéral légèrement concave.

Longueur : $0,254(0,210-0,275)$.

Largeur : $0,317(0,294-0,325)$.

Quatre paires de soies scutales: $\mathrm{Ss}_{1} \ldots \mathrm{Sc}_{4}$.

\section{Coxae et Tarse I.}

Coxa I. Un éperon interne. Un éperon postéroexterne inconstant. Troies soles.

Coxa II. Une crête externe et une interne. Deux soies.

Coxa III. Légère saillie externe et interne. Deux soies.

Longueur du tarse I : $0,117(0,160-0,220)$.

\section{Chétotaxie Générale.}

Face ventrale.

Trois paires de soies sternales: $\mathrm{St}_{1}, \mathrm{St}_{2}, \mathrm{St}_{3}$. Longueur des soies: $\mathrm{St}_{1}: 0,034$ $(0,028-0,040) ; \mathrm{St}_{2}: 0,021(0,018-0,026) ; \mathrm{St}_{3}: 0,023(0,016-0,035)$.

Distances séparant $\mathrm{St}_{1}$ et $\mathrm{St}_{1}, \mathrm{St}_{1}$ et $\mathrm{St}_{3}: \mathrm{St}_{1}-\mathrm{St}_{1}: 0,045(0,034-0,051) ; \mathrm{St}_{1}-\mathrm{St}_{3}$ : 0,021 (0,104-0,154).

Trois paires de soies préanales: $\mathrm{Pa}_{1}, \mathrm{~Pa}_{2}, \mathrm{~Pa}_{3}$.

Sept paires de soies prémarginales: $\mathrm{Pm}_{1} \ldots \mathrm{Pm}_{7}$. Longueur de $\mathrm{Pm}_{7}: 0,039$ $(0,035-0,049)$.

Trois paires de soies marginoventrales: $\mathrm{Mv}_{1}, \mathrm{Mv}_{2}, \mathrm{Mv}_{3}$.

Face dorsale.

Quatre paires de soies centrodorsales: $\mathrm{Cd}_{1} \ldots \mathrm{Cd}_{4}$.

Quatre paires de soies supplémentaires: $S_{1} \ldots S_{4} . S_{1}: 0,046(0,043-0,051) ; S_{4}$ : $0,041(0,031-0,049)$.

Huit paires de soies marginodorsales: $\mathrm{Md}_{1} \ldots \mathrm{Md}_{\mathrm{s}} . \mathrm{Md}_{1}$ : $0,057(0,052-0,063)$; $\mathrm{Md}_{8}: 0,030(0,024-0,036)$. 


\section{Clef des Larves du Genre Ixodes*}

1. - Article I des palpes plus ou moins hypertrophié et irrégulier avec une ou deux saillies

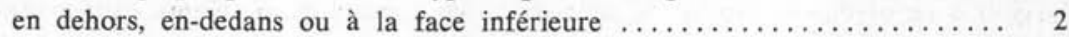

Article I des palpes peu ou pas hypertrophié, ne présentant de saillie ni en dedans,

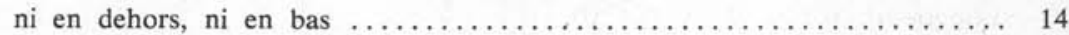

2. - Premier article avec deux prolongements, l'un antéro-interne, l'autre postéro-externe.

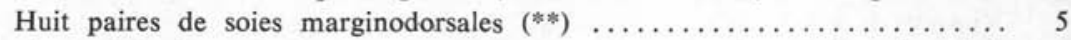

Premier article des palpes avec un prolongement interne très développé, parfois une courte saillie arrondie du côté externe mais pas de vrai prolongement. Pas d'auricules. Six paires de soies marginodorsales $\ldots \ldots \ldots \ldots \ldots \ldots \ldots \ldots \ldots, 3$

Premier article des palpes avec un prolongement postéroexterne ou une saillie ventrale. Huit ou neuf paires de soies marginoventrales $(* *) \ldots \ldots \ldots \ldots \ldots .10$

3. - Hypostome avec $2 / 2$ files de dents. Ecusson : $0,35 / 0,45 \ldots \ldots \ldots \ldots \ldots \ldots$ Hypostome $2 / 2+3 / 3$. Ecusson $0,20 / 0,30$. Tarse $I$ : 0,150 :

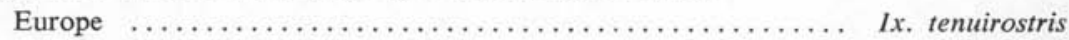

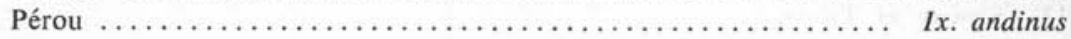

4. - Palpes II + III : 0,16. Tarse I : $0,270(11,25) \ldots \ldots \ldots \ldots \ldots$ Ix. trianguliceps Palpes II + III : 0,260 . Tarse I : $0,260 \ldots \ldots \ldots \ldots \ldots \ldots \ldots \ldots \ldots \ldots \ldots \ldots \ldots \ldots \ldots$ nuttalli Palpes II + III : 0,20 . Afr. orientale et méridionale $(70) \ldots \ldots \ldots$ Ix. alluaudi

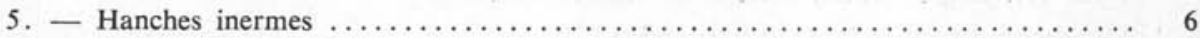
Hanches quelquefois pourvues d'épines plus ou moins marquées $\ldots \ldots \ldots \ldots \ldots 7$

6. - Hypostome à $2 / 2$ files de 8 dents. Sillon cervical bien marqué, atteignant le bord

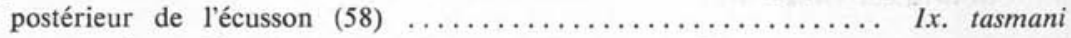

Hypostome à $2 / 2$ files de 5 dents. Sillon cervical mal défini, n’atteignant pas le bord

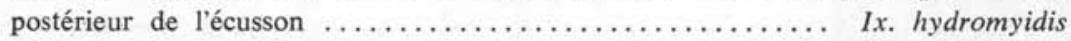

7. - Ecusson un peu plus long que large $\mathrm{L} / 1$ : 1,09. Quatre paires de soies marginoventrales. Soies posthypostomales A-A' environ égales à P-P' (1) $\ldots \quad I x$. sculptus

Ecusson un peu plus large que long $\mathrm{L} / 1$ : 0,70 à 0,80 . Trois paires de soies marginoventrales. A-A' nettement plus grand que P-P' ................ 8

8. - File externe des dents de l'hypostome à $6 / 7$ dents. File 2 à 8 dents ....... 9 File externe 1 à 6 dents. File 2 à 6 dents. Indice du palpe $\mathrm{II}+\mathrm{III} / 1$. : 2,7 Ix. stromi

9. - Indice du palpe II + III/1.: 2,8. Soies marginodorsales: 0,024 à 0,030 en

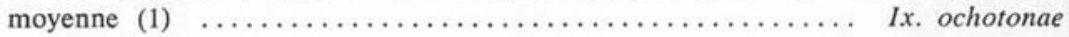

Indice du palpe II + III/1.: 1,8. Soies marginodorsales: 0,045 en moyenne (1)

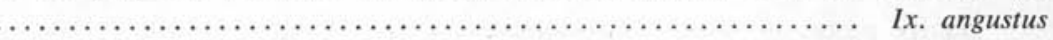

(*) Comme la précédente, cette clé est donnée sous toute réserve. Nul doute que des travaux ultérieurs permettront de la rectifier et de la compléter. Les chiffres entre parenthèses, rencontrés çà et là, renvoient aux travaux où le caractère a été étudié. Se reporter à la note précédente : Ann. Parasitologie hum. et comp., 1966.

(**) Ce caractère n'a pu être étudié chez diverses espèces. 
10. - Bord postérieur de l'écusson cordiforme. Maximum de largeur tout à fait en

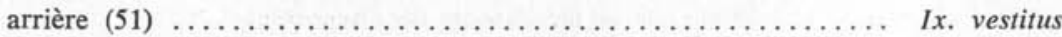

Bord postérieur de l'écusson tout à fait anguleux. Maximum de largeur vers la partie moyenne. Bords postérolatéraux de l'écusson distincts, légèrement échan-

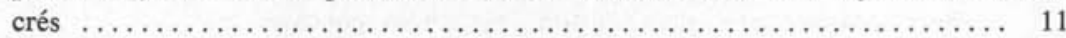

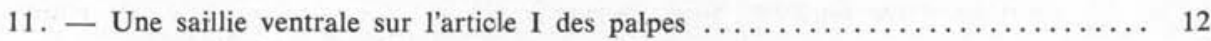

Une projection postéroexterne sur ce même article $\ldots \ldots \ldots \ldots \ldots \ldots \ldots \ldots \ldots$

12. - Trois à quatre paires de soies marginoventrales. Epine interne de la hanche I arron-

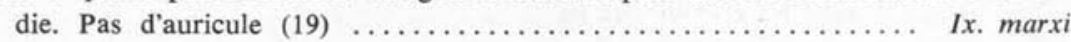

Quatre paires de soies marginoventrales. Epine interne de la hanche I bien mar-

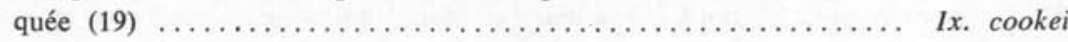

13. - Distance entre les soies posthypostomales postérieures à peu près égale à celle entre

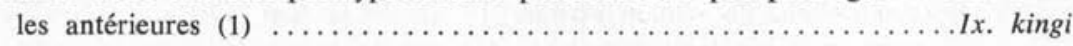
Soies posthypostomales postérieures plus rapprochées que les antérieures (1)

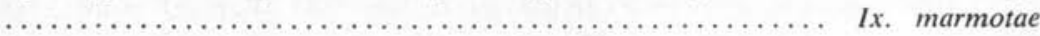

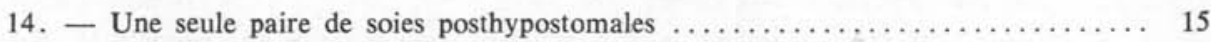

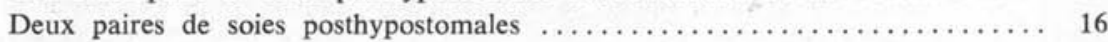

15. - Dentition $2 / 2$. Indice du capitulum $\mathrm{L} / 1$ : 1 . Indice des palpes $\mathrm{II}+\mathrm{III} / 1$. : 2 (11)

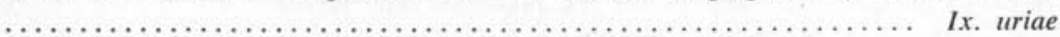

Dentition $3 / 3$. Indice du capitulum $\mathrm{L} / 1: 0,26$. Indice des palpes $\mathrm{II}+\mathrm{III} / 1$.: 1,5

Ix. paradoxus

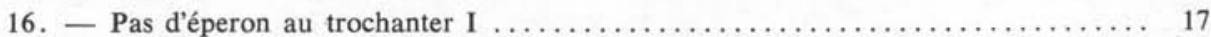

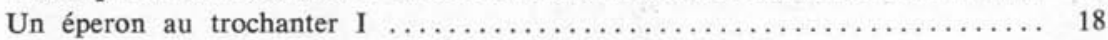

17. - Pattes longues. Tarse I supérieur à $0,35(11,25) \ldots \ldots \ldots \ldots$ Ix. vespertillionis Pattes moyennes ou courtes. Tarse $I$ inférieur ou égal à $0,26 \ldots \ldots \ldots \ldots 23$

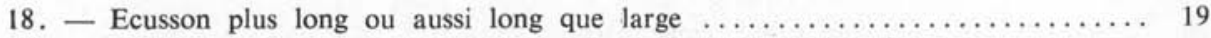

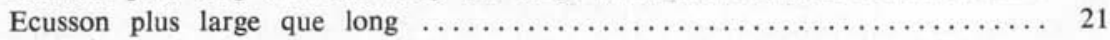

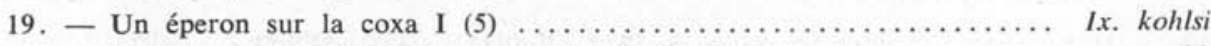

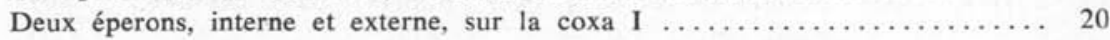

20. - Sillon anal formé, circulaire $(12) \ldots \ldots \ldots \ldots \ldots \ldots \ldots \ldots \ldots \ldots \ldots$. muniensis Sillon anal ouvert en arrière $\ldots \ldots \ldots \ldots \ldots \ldots \ldots \ldots \ldots \ldots \ldots \ldots \ldots \ldots \ldots$. rotschilddi

21. - Pattes longues. Tarse I égal ou supérieur à $0,35 \ldots \ldots \ldots \ldots \ldots \ldots \ldots \ldots \ldots \ldots \ldots \ldots \ldots \ldots \ldots$ Pattes plus courtes et plus trapues. Tarse I inférieur à 0,35 (11) $I x$, caledonicus

22. - Auricules fortes, bien marquées $(25) \ldots \ldots \ldots \ldots \ldots \ldots \ldots \ldots \ldots \ldots \ldots \ldots \ldots$ Ix. berlesei Auricules à peine ébauchées. Grande Bretagne (11) $\ldots \ldots \ldots \ldots$ Ix. unicavatus Auricules à peine ébauchées. Crimée $(25) \ldots \ldots \ldots \ldots \ldots \ldots \ldots \ldots \ldots \ldots \ldots$ tauricus

23. - Soie supplémentaire unique ou absente. Quatre soies prémarginales $\ldots \ldots \ldots 26$ Quatre soies supplémentaires. Six à sept soies prémarginales ............ 24

24. - Longueur voisine de $0,5 \mathrm{~mm}$. Palpes allongés et minces. Rapport palpe/capitulum $=1$. Hypostome à apex arrondi. Dix à douze dents sur la file externe de

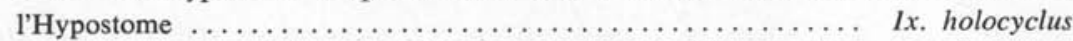
Longueur du corps inférieure à $0,45 \mathrm{~mm}$. Palpes claviformes. Rapport palpe/capiAnnales de Parasitologie humaine et comparée (Paris), t. 42, 1967, $\mathrm{n}^{\circ} 1$ 
tulum nettement inférieur à 1 . Apex de l'hypostome plat ou légèrement excavé. Sept à huit dents sur la file externe de l'hypostome $\ldots \ldots \ldots \ldots \ldots \ldots 25$

25. - Bord postérolatéral de l'écusson convexe. Absence d'auricules. Soies courtes.

Bord postérolatéral de l'écusson légèrement concave.

Présence d'une auricule. Soies longues $\ldots \ldots \ldots \ldots \ldots \ldots \ldots \ldots$ Ix. pilosus

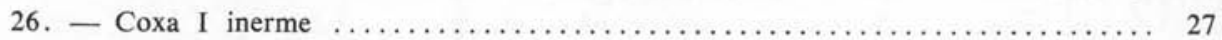

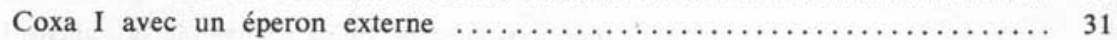

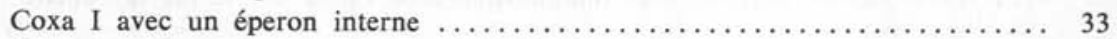

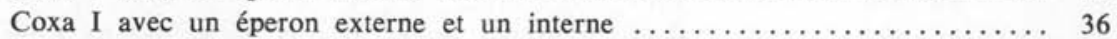

27. - Scutum avec un bord postérieur et deux bords postérolatéraux plus ou moins

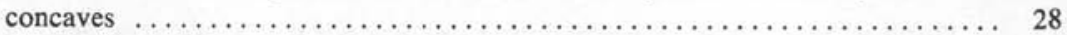

Bord postérieur du scutum arrondi. Indice du scutum 0,90 .

Rapport palpe/capitulum 1,34. Arkansas. (29) ............. Ix. banksi

Bord postérieur arrondi. Indice du scutum 0,84. Rapport palpe/ capitulum 1,07. Australie, Tasmanie, Mariannes (58) ............. Ix. ornithorhynchi

Bord postérieur arrondi. Indice du scutum 0,91. Rapport palpe/capitulum 0,71. Iles

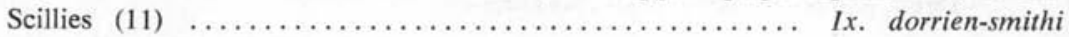

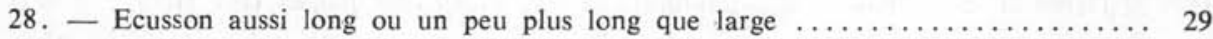

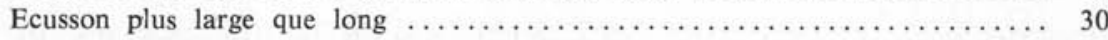

29. - Un étranglement très net sur la partie postérieure du bord latéral de la face ven-

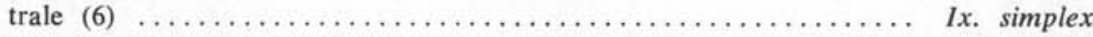
Pas d'étranglement semblable $(11) \ldots \ldots \ldots \ldots \ldots \ldots \ldots \ldots \ldots \ldots \ldots \ldots \ldots \ldots$ lividus

30. - Index du capitulum 1,6. Chien, Renard, Blaireau. Europe ...... Ix. canisuga Index du capitulum 1,1. Oiseaux d'Europe septentrionale (11) $\ldots l x$. arboricola Index du capitulum 1,8, Europe orientale. Asie (25) $\ldots \ldots \ldots \ldots$ Ix. crenulatus

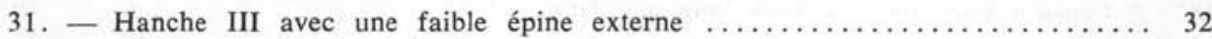
Hanche III inerme $(58) \ldots \ldots \ldots \ldots \ldots \ldots \ldots \ldots \ldots \ldots \ldots \ldots \ldots \ldots \ldots \ldots \ldots \ldots \ldots$, trichosuri $(*)$

32. - Hypostome $2 / 2$, non rétréci à la base. Rapport palpe/capitulum: $0,096 / 0,15=$

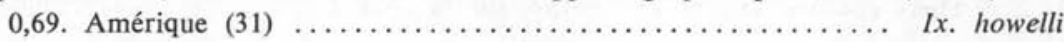
Hypostome 2/2, rétréci à la base. Rapport palpe/capitulum 0,62. Amérique du

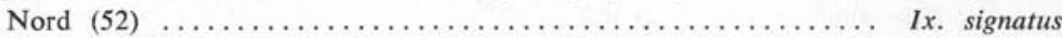

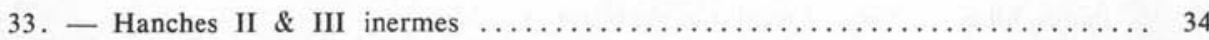
Hanches II \& III avec des ébauches d'éperons internes et externes. Indice du palpe supérieur à 3,5. Deux paires de soies centrodorsales ......... Ix. bishoppi

34. - Face ventrale ressérée en arrière de la moitié $(3,11) \ldots \ldots \ldots \ldots$ Ix. hexagonus Face ventrale avec bords postérolatéraux régulièrement arrondis. Deux paires de centrodorsales. Huit ou neuf paires de marginodorsales ........ Ix. texanus

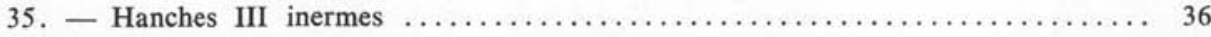

Hanches III avec un ou deux éperons plus ou moins nets $\ldots \ldots \ldots \ldots \ldots \ldots, 41$

(*) Ix. vanidicus Schulze se classerait ici avec Ix. trichosuri ou avec le groupe Howelli-holocyclus, selon que la hanche est inerme ou munie d'un éperon, ce que Schulze n'indique pas. 


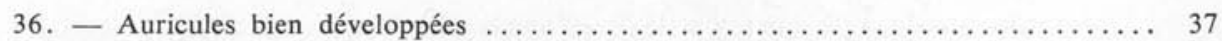

Auricules réduites à des crêtes plus ou moins distinctes $\ldots \ldots \ldots \ldots \ldots \ldots \ldots . \ldots \ldots$

37. - Indice des palpes II + III/L. : 2,6. U.S.A. \& Canada (1) . .... Ix. pacificus

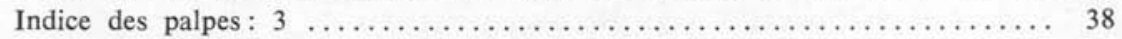
Indice des palpes: 4. Afrique centrale (9) $\ldots \ldots \ldots \ldots \ldots \ldots$ Ix. schillingsi

38. - Rapport longueur du capitulum/longueur du palpe: 2,5 .

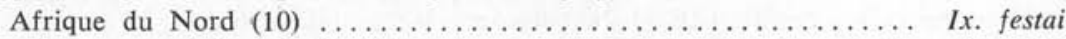

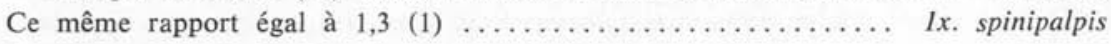

39. - Espèce Sud-américaine $(34) \ldots \ldots \ldots \ldots \ldots \ldots \ldots \ldots \ldots \ldots \ldots \ldots \ldots \ldots \ldots \ldots \ldots$ Ix amarali $\left({ }^{*}\right)$ Espèces européennes, russes, africaines, nord-américaines, Panama ...... 40

40. - Coxa II inerme. Soie $\mathrm{Md}_{1}$ égale, en moyenne, à 0,059 (3) . . . . . Ix. tiptoni Coxa II avec un tubercule interne et une petite saillie externe. Soie $\mathrm{Md}_{1}: 0,036$ (19)

Ix. muris

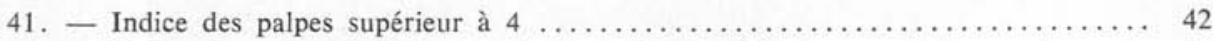

Indice des palpes inférieur ou tout au plus égal à $4 \ldots \ldots \ldots \ldots \ldots \ldots \ldots \ldots$

42. - Indice supérieur à 5. Tarse I court. Madagascar (7) $\ldots \ldots \ldots$ Ix. colas-belcouri Indice compris entre 4 et 5 . Tarse I long et aminci.

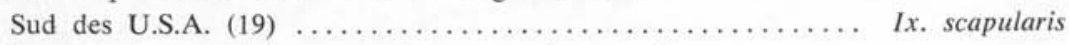

Indice entre 4 et 5 . Epine assez longue sur la coxa $\mathrm{I}$.

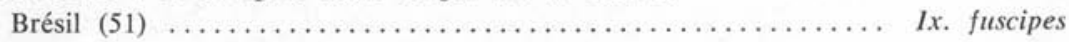

43. - Epine interne de la hanche supérieure à l'externe $\ldots \ldots \ldots \ldots \ldots \ldots \ldots \ldots \ldots$

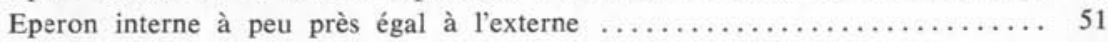

Eperon interne plus petit que l'externe. Pas de cornuæ $(58) \ldots \ldots$ Ix. eudyptidis

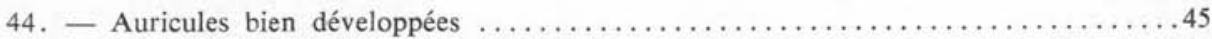
Auricules absentes ou peu visibles (19) $\ldots \ldots \ldots \ldots \ldots \ldots \ldots \ldots \ldots \ldots \ldots \ldots \ldots$ Ix , dentatus

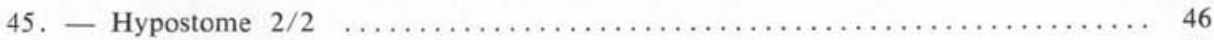

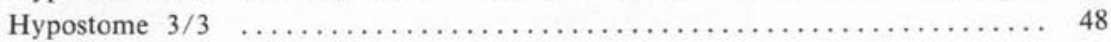

46. - Soies antérieures de lalloscutum égales à $2 \& 3$ fois la longueur des soies scu-

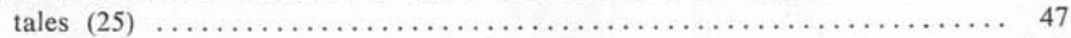
Ces mêmes soies égales à 1,4 fois les soies scutales $(25) \ldots \ldots \ldots$ Ix. occultus

47. - Soies alloscutales en bâtonnets $(25) \ldots \ldots \ldots \ldots \ldots \ldots \ldots \ldots \ldots \ldots \ldots \ldots \ldots \ldots \ldots \ldots \ldots \ldots$ Ix. laguri Ces mêmes soies aculéiformes $(25) \ldots \ldots \ldots \ldots \ldots \ldots \ldots \ldots \ldots \ldots \ldots \ldots \ldots$ Ix. redikorzevi

48. - Longueur de l'écusson égale à sa largeur $(25) \ldots \ldots \ldots \ldots \ldots$ Ix. kazakstani Longueur de l'écusson inférieure à sa largeur .................... 49

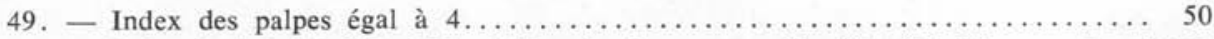
Index égal à 3,5 . Soie $\mathrm{Md}_{1} 4$ fois plus longue que $\mathrm{Sc}_{3}(21,25,67) \ldots$ Ix. ricinus Index égal à 3,5 . Soie $\mathrm{Md}_{1} 3$ fois plus longue que $\mathrm{Sc}_{3} \ldots \ldots \ldots$ Ix. persulcatus

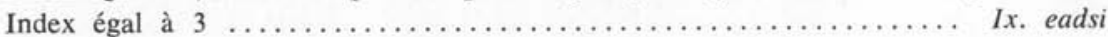

50. - Bord postéroventral du capitulum ondulé. Angleterre, Europe septentrionale, Rus-

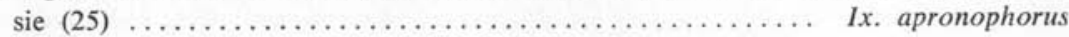

(*) La description originale de Fonseca ne permet pas d'utiliser un autre caractère différentiel. 
Bord postéroventral du capitulum légèrement convexe.

Capitulum étranglé à la base. Etats-Unis (29) $\ldots \ldots \ldots \ldots \ldots \ldots$ Ix. minor

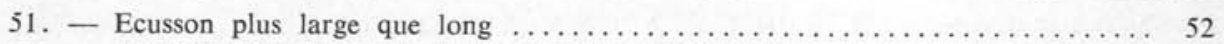

Ecusson plus long que large. Hypostome $2 / 2(51) \ldots \ldots \ldots \ldots \ldots$ Ix. loricatus

52. - Indice des palpes 2,9. Article II nettement rétréci à la base.

Eperon interne court sur la coxa I. Europe $(11) \ldots \ldots \ldots \ldots \ldots \ldots$. part Indice des palpes 4 . Base du palpe peu nettement rétrécie.

Epine interne de la coxa $\mathrm{I}$ longue Etats-Unis (19) .......... Ix. brunneus (Ix. cumulatim punctatus) semble se rapprocher de ce groupe).

(Laboratoire de Parasitologie de la Faculté de Médecine d'Alger et de la Faculté de Médecine de Paris 